Fecha de recepción: marzo 2021 Fecha de aprobación: abril 2021 Fecha publicación: mayo 2021

\section{Diseño para la eco-innovación: hacia un mejor escenario futuro basado en el alineamiento de la investigación impulsada por el diseño}

Katherine Mollenhauer ${ }^{(1)}$ y Bernardo Antonio Candela Sanjuán ${ }^{(2)}$

Resumen: Actualmente no se entiende un proceso de innovación que no tenga en cuenta el impacto económico, social y medioambiental que puede derivar de su implementación. El diseño como agente de innovación de las prácticas socioculturales, puede facilitar el cambio y la transformación necesaria para que el futuro sea sostenible, justo y próspero para el planeta y sus comunidades. Sin embargo, la identificación del aporte del diseño, requiere determinar los principios, criterios y componentes metodológicos que deben ser activados, y ponerlos en acción mediante un método que aborde el proceso co-creativamente, desde la identificación del problema u oportunidad, la ideación de las posibles respuestas de solución hasta la adjudicación de fondos concursables para la implementación del proyecto.

La investigación se realizó en el contexto de un programa de cooperación internacional de investigación, financiado por instituciones públicas y privadas, que generó propuestas para proyectos de investigación impulsados por el diseño, alineados específicamente con el eje prioritario "Crecimiento verde y sostenibilidad" de la Estrategia de Especialización Inteligente de Canarias 2014-2020. Los autores sistematizaron los resultados desde una perspectiva de investigación aplicada y de diseño de caso único y como resultado presentan una metodología y un toolkit para el alineamiento de la investigación impulsada por el diseño con las políticas territoriales de investigación e innovación.

Finalmente, los autores a partir de los resultados de la aplicación, concluyen que el desarrollo de una metodología que permita la alineación y active los potenciales aportes del ecosistema de diseño con un sistema de innovación territorial determinado, tiene como beneficios ampliar las oportunidades de acción de la disciplina, al mismo tiempo que aportar al logro de los objetivos estratégicos para el desarrollo sostenible de la región.

Palabras clave: Diseño para la Eco-Innovación - Ecosistema Diseño - Metodología de Diseño - Políticas de Innovación guiadas por Diseño - Metodología Participativa - Ecosistema de Innovación Local - Enfoque Sistémico.

[Resúmenes en inglés y portugués en las páginas 277-279] 
${ }^{(1)}$ Katherine Mollenhauer, por la Pontificia Universidad Católica de Chile (Chile). Es Doctora en Diseño Estratégico e Innovación por la Universidad de Barcelona, Doctora Europeus por el Politecnico di Milano y Aalto University, Máster en Problemáticas Formales del Producto por la Universitat Jaume I de Castellón y Diseñadora (m) Equipamiento por la Universidad Tecnológica Metropolitana. Imparte docencia en el Grado de Diseño y en el Magister en Diseño Avanzado de la Facultad de Arquitectura, Diseño y Estudios Urbanos de la Pontificia Universidad Católica de Chile (PUC). Su investigación está centrada, por una parte, en el rol, uso y aporte de modelos y metodologías de diseño de servicio en la relación usuario-políticas públicas-servicios públicos aplicados a la promoción al Diseño en Sistemas de Innovación Nacional y al (re)diseño de servicios públicos. Y por otra, en el desarrollo sostenible de comunidades y territorios mediante modelos y metodologías de innovación Design-Driven aplicados al diseño de productos, servicios y experiencias para el desarrollo local, del territorio y de sectores productivos. Es miembro del Laboratorio de Innovación Pública (LIP), del Grupo de Diseño de Servicios (DSUC) y del Núcleo de Gobernanza y Ordenamiento Territorial (NUGOT) de la PUC.

(2) Bernardo Antonio Candela Sanjuán, por la Universidad de La Laguna (España). Es Doctor en Bellas Artes por la Universidad de La Laguna (ULL), Ingeniero Técnico en Diseño Industrial por la Universidad Politécnica de Valencia, Máster en Diseño y Fabricación por la Universitat Jaume I de Castellón y Máster en Innovación en Diseño para el sector turístico por la Universidad de La Laguna. Imparte Docencia en el Grado de Diseño de la Facultad de Bellas Artes y en el Máster en Innovación en Diseño para el Sector Turístico de la Universidad de La Laguna. Su investigación está centrada en el estudio de la relación entre Diseño y Política, trabajando en temas vinculados a las políticas de diseño, al diseño de políticas, la participación ciudadana y la innovación. Es miembro del Grupo de Investigación e Innovación en Diseño de la ULL.

\section{Introducción}

En los últimos años, la Unión Europea ha alentado a sus Estados miembros a incorporar el diseño en las políticas de innovación nacionales y regionales. El Plan de acción para la innovación impulsada por el diseño (Comisión Europea, 2013) incluye entre sus objetivos: "promover la innovación impulsada por el diseño en las industrias para fortalecer la competitividad de Europa" (p. 8), y "promover nuevas estrategias y prácticas de innovación colaborativa que permitan nuevos modelos de negocio" (p. 9). De acuerdo con Whicher (2017) la innovación impulsada por el diseño juega un papel clave en el crecimiento y desarrollo de la UE.

En este contexto, el diseño surge como un agente con potencial de innovación para la creación de soluciones sostenibles que facilitan el cambio social y medioambiental necesario para un futuro sostenible, justo y próspero para las personas y el planeta. Sin embargo, para que el diseño promueva procesos de eco-innovación es necesario determinar cuáles 
son los principios y criterios que deben ser puestos acción, así como cuáles son los medios o componentes que pueden operacionalizar la generación de nuevos escenarios futuros sostenibles, justos y prósperos para la humanidad y el medio ambiente.

Los autores defienden, que si bien el diseño ha logrado insertarse en diversos espacios de las estrategias de innovación nacionales y regionales, aún son escasos los métodos e instrumentos que permitan una participación efectiva de los sistemas diseño, en particular, en los ámbitos relacionados con la eco-innovación. Por lo tanto

- ¿cuáles son los principios y criterios que pueden impulsar un procesos de eco-innovación guiado por diseño?

- ¿qué componentes deben estar presentes en un proceso de eco-innovación guiado por diseño?

- ¿cómo deben ser puestos en acción el conjunto de principios, criterios y medios para que proceso de eco-innovación guiado por diseño puedan ser impulsados?

La respuesta a estas preguntas se responden desde el Diseño para la Transición, como un enfoque que integra las interrelaciones entre los sistemas naturales, sociales y económicos (Irwin, 2015), para abordar el desarrollo de soluciones a problemas que surgen en contextos complejos. De acuerdo a Spencer, el diseño por su "enfoque único de resolución de problemas: está centrado en las personas, es impulsado por las posibilidades, está enfocado en las opciones y es iterativo" (Spencer, 2018, p. 91).

Por lo tanto, la creación de un método para la alineación de los potenciales aportes del ecosistema de diseño local con los objetivos estratégicos de desarrollo sostenible de un sistema de innovación territorial crea nuevas sinergias entre los actores y su entorno. La definición de los principios, criterios y componentes claves que deben estar presentes en la formulación de este tipo de proyectos, facilitan la transición hacia una economía circular y amplía las oportunidades de acción de la disciplina del diseño.

\section{Marco teórico}

Junto con la exposición de una experiencia de investigación aplicada que permita contextualizar la práctica del diseño en la construcción metodológica y funcional para el alineamiento de la investigación impulsada por el diseño con las políticas territoriales de investigación en innovación, es importante presentar algunos conceptos que enmarcan el ámbito de actuación de dicha propuesta.

El diseño facilita el cambio, es un agente que permite soluciones sostenibles para las personas en todos los aspectos de la sociedad, y del planeta del que dependemos, para la transición hacia una mejor calidad de vida (World Design Summit Organization Inc. 2017). El diseño puede contribuir a generar procesos participativos que sean más conscientes y que activen a las comunidades, a las organizaciones y/o a las administraciones públicas. El diseño ha pasado de una perspectiva habitual centrada en el producto y su comercialización, a otra enfocada en la activación de procesos y nuevas metodologías. Y es desde ahí, desde la resolución de problemas que afectan a la sociedad, de los problemas sociales, 
desde donde acontecen valores intrínsecos al diseño: la participación, la investigación, y la mejora del entorno.

A continuación se presentan una serie de conceptos y antecedentes que sustentan el caso presentado.

\section{a. Ecosistema Diseño}

La teoría de los "Sistema Nacionales de Innovación" de finales del siglo XX de Lundvall (1988) y Freeman (1995) fue adaptada a principios del siglo XXI por académicos e investigadores para proporcionar argumentos a la integración del diseño en las políticas gubernamentales de innovación. De acuerdo con Whicher (2016), a pesar de que el diseño fue un factor en el concepto primario del Sistema Nacional de Innovación presentado por Freeman a la Organización para la Cooperación y el Desarrollo Económico (OCDE) en el año 1982, las dificultades de medir su impacto hizo que fuera olvidado por los posteriores teóricos de la innovación.

La terminología utilizada para definir la teoría de ecosistemas de diseño ha sido variada, lo que no ha permitido un buen asentamiento teórico del concepto. Los términos utilizados que hacen referencia a este concepto son:

- Design infrastructure (Love, 2005), Sistema de Innovación Design_Driven (Mollenhauer, 2007), National Design System (Moultrie y Livesey 2008, Raulik-Murphy and Cawood 2009, Ministerio de Comercio, Industria y Turismo del Gobierno de Colombia 2009, Sun 2010, Swann 2010, Alarcón 2011, Hobday et al. 2012, Whicher y Cawood 2012); - System dynamic of national design infrastructure (Love, 2007), Dynamic Design Ecosystem (Ministerio de empleo y economía de Finlandia 2014) y

- Design Innovation Ecosystem (Whicher 2014, Chisol et al. 2013).

En la actualidad, ya existe un consenso entre la comunidad académica y científica para utilizar el término «Ecosistema Diseño». Se basa en la lógica de que se necesita la interacción compleja de actores de diversa naturaleza y de acciones dentro de un territorio delimitado. Además, estas iniciativas pueden darse entre iguales sin la necesidad de jerarquías entre tipos de actores, por ello se descarta nombrarlo sistema. El concepto de ecosistema es interdependiente, por ello, cualquiera aspecto debe analizarse dentro de su contexto como un todo (Whicher et al., 2017).

El concepto Ecosistema de Diseño es un modelo que reúne los actores, el medio ambiente, las estructuras y sus interrelaciones para apoyar el diseño como impulsor de la innovación en un territorio (nacional o regional). Este modelo permite detectar sus fortalezas y debilidades, y ayudar a la formulación de políticas de diseño e innovación (Chisolm, Mortati y Villari, 2013). Un ecosistema de diseño es un marco de análisis para la mejora de los ecosistemas de innovación de un país o una región.

Desde el año 2010, el diseño es reconocido en Europa como factor de competitividad, desarrollo, crecimiento y prosperidad gracias a su integración en las políticas europeas de innovación por medio de la Unión por la Innovación -iniciativa insignia de la Estrategia 
para el crecimiento Europa 2020-, de este modo se valora al diseño como fuerza motora para la transformación de las empresas, el sector público y la sociedad. En el año 2013, este compromiso fue materializado con el lanzamiento del Plan de Acción para la Innovación impulsada por el diseño que tiene como objetivo acelerar la adopción del diseño en la política de innovación nacional y regional. Este documento reconoce que:

Un uso más sistemático del diseño como herramienta para la innovación centrada en el usuario y orientada al mercado en todos los sectores de la economía, complementaria a la I+D, mejoraría la competitividad europea y el desarrollo social y cultural de un territorio (Comisión Europea, 2013, p. 4).

En la actualidad, la nueva agenda estratégica del Consejo Europeo para 2019-2024 se centra en cuatro prioridades principales, en torno a la protección de la libertad cívica, garantizando economía, construyendo sociedades climáticas neutrales y justas, y promoviendo intereses y valores europeos a nivel mundial.

Tras el éxito de varias iniciativas de diseño de la Comisión Europea, incluida una Acción Plan para la innovación basada en el diseño, BEDA (Bureau European Designs Associations $)^{1}$ está trabajando para dar forma a la próxima generación de políticas de diseño para Europa. Por ello, esta organización ha identificado cuatro grandes ámbitos de acción del Diseño ${ }^{2}$ alineados con aquellas Prioridades de la Comisión Europea para mostrar cómo aprovechar las potencialidades del diseño para impulsar la innovación y el crecimiento de la economía, liderada por la transformación ecológica basada en la Eco-Innovación.

\section{b. Eco-Innovación}

La literatura, por el momento, no ofrece una definición clara y consensuada sobre el término Eco-Innovación. Faltan evidencias empíricas necesarias para construir un marco conceptual y teórico adecuado (Álvarez et al., 2014), aunque es cierto que diferentes organismos internacionales demandan "enverdecer" las economías e introducir la eco-innovación como una herramienta para ese cambio (Comisión Europea 2011, OCDE 2014, Unión Europea 2010). Es tal la importancia dotada a la eco-innovación en Europa que cuenta con un Plan de Acción sobre Eco-innovación que es "el corazón de las políticas europeas" ("El Plan de Acción sobre Eco-Innovación - Plan de Acción sobre Eco-Innovación - Comisión Europea”, 2020).

En una reciente revisión de la literatura, O’Ryan y Schaper (2017) señalan que según Díaz-García C. et al. (2015)

(...) se han acuñado cuatro conceptos relacionados con la innovación que buscan reducir el daño ambiental: innovación verde, innovación ambiental, innovación sostenible y eco-innovación.

Según los autores, apoyándose en los estudios de Schiedering et al. (2012), indican que todas las definiciones comparten las mismas características y se utilizan indistintamente, 
salvo la eco-innovación, que agrega de manera explícita la necesidad de considerar el enfoque de ciclo de vida en el análisis sobre el impacto ambiental. Un aspecto importante para el concepto de eco-innovación es la reducción del impacto ambiental (Johansson y Magnusson 1998, Rennings 2000, Kuehne 2007, Unión Europea 2007). Todas las definiciones surgidas hasta el 2007 estaban vinculadas a los productos, las técnicas y los procesos, pero con la aparición del marco de Competitividad e Innovación de la Unión Europea (2007) surgen nuevas definiciones inclusivas vinculadas al "desarrollo sostenible", a la incorporación del cambio organizativo como factor de eco-innovación y a la intencionalidad o no de la misma (OCDE 2009, Machiba 2010, Halila y Rundquist 2011 y Carrillo-Hermosilla et al. 2010).

No es hasta el 2010 cuando la literatura incorpora definitivamente el enfoque del "Ciclo de Vida" en su definición, volviéndola más precisa, p.ej. el Observatorio de Eco-Innovación de la Unión Europea (2010) construye una definición del concepto de eco-Innovación a partir de las propuestas de la OCDE (2009) y la Comisión Europea (2010) con el sustento de la definición del Manual de Oslo desde un enfoque de ciclo de vida y definiendo la eco-innovación como:

(...) la introducción de cualquier producto nuevo o significativamente mejorado (bien o servicio), proceso, cambio organizativo o solución de marketing, que reduce el uso de recursos naturales (incluidos los materiales, energía, agua y tierra) y disminuye la liberación de sustancias nocivas a lo largo de su ciclo de vida (p. 4).

En conclusión, se trata de una definición integradora, no excluyente que permite diferenciar diferentes tipos de Eco-Innovación (Vicente y Tamayo, 2014):

- Eco-Innovación de procesos

- Eco-Innovación de productos

- Eco-Innovación en la organización

- Eco-Innovación de comercialización

- Eco-Innovación social, y

- Eco-Innovación en el flujo de materiales.

\section{c. Estrategias Inteligentes de Especialización guiadas por diseño}

En diciembre del año 2013, el Consejo de la Unión Europea aprobó las normas que rigieron la ronda de inversión de la política de cohesión de la Unión Europea para el período 2014-2020. Estas directrices establecieron el desarrollo de las conocidas como RIS3 por sus siglas en inglés (Research and Innovation Strategies for Smart Specialization). Las Estrategias de Investigación e Innovación para la Especialización Inteligente (RIS3) fueron un requisito previo de la Comisión Europea, a los países y regiones de la UE para el acceso a los fondos europeos en los ámbitos de investigación, innovación y sociedad de la infor- 
mación hasta el año 2020. En el documento de la Comisión $\operatorname{COM(2010)} 553$ final, esta estrategia se define de la siguiente manera:

(...) En esencia, la especialización inteligente trata de poner mayor énfasis en la innovación y en concentrar los escasos recursos humanos y financieros de $\mathrm{I}+\mathrm{D}+\mathrm{i}$ en unas pocas áreas competitivas globalmente (p. 41).

Este hecho supone un cambio de enfoque en relación a las políticas de innovación que se habían realizado hasta el momento basadas en los planteamientos, que con objeto de favorecer el desarrollo y disminuir las diferencias interterritoriales, las políticas deben ser neutrales desde el punto de vista territorial. No obstante, teniendo en cuenta que el contexto varía notablemente de unos territorios a otros, supone una gran oportunidad para la definición de una estrategia de desarrollo del sistema de I+D+i local teniendo en cuenta sus características propias y aquellos aspectos en lo que pueden tener ventajas y mayor potencial en relación a otras regiones europeas y mundiales, con el fin de evitar enfoques o políticas miméticas entre regiones.

Por consiguiente, la Estrategia de Especialización Inteligente de Canarias 2014-2020 es un documento de planificación de la política de $\mathrm{I}+\mathrm{D}+\mathrm{i}$ del Gobierno de Canarias que tiene como objetivos destacados la coordinación y potenciación del sistema regional de $\mathrm{I}+\mathrm{D}+\mathrm{i}$, lograr un uso más eficaz de los fondos públicos y estimular la inversión privada en $\mathrm{I}+\mathrm{D}+\mathrm{i}$. Las prioridades temáticas de la RIS3 para Canarias fueron consensuadas con representantes de todos los ámbitos de la región, esto es, las administraciones públicas, los centros de conocimiento (universidades y centros de investigación), las empresas y asociaciones empresariales y la sociedad.

En el estudio de caso se relacionan las cinco prioridades definidas en la Estrategia de Especialización Inteligente (RIS3) de Canarias para el periodo 2014-2020:

I. Liderazgo inteligente del turismo

II. Canarias, referente atlántico inteligente

III. Valorización socioeconómica de la $\mathrm{I}+\mathrm{D}$, especialización y fortalecimiento en astrofísica y espacio, ciencias marítimo-marinas, biotecnología y biomedicina asociadas a la biodiversidad y enfermedades tropicales

IV. Agenda Digital y

V. Crecimiento verde y sostenibilidad

\section{Metodología para una investigación a través del diseño}

La investigación se realizó en el contexto del proceso de ideación y desarrollo de un taller bajo un enfoque sistémico y de co-creación que contó con la participación de un grupo de actores que hacen parte del ecosistema de innovación en diseño de Canarias (España), compuesto por investigadores, organizaciones de miembros de la industria, consultores 
estratégicos, instituciones gubernamentales y diseñadores. El propósito del taller estuvo relacionado con la identificación de propuestas para proyectos de investigación impulsados por el diseño alineados específicamente con el eje prioritario "Crecimiento verde y sostenibilidad" de la Estrategia de Especialización Inteligente de Canarias 2014-2020.

Mediante un proceso de investigación aplicada, específicamente desde la investigación-acción se logró identificar aquellos componentes y el proceso mediante el cual es posible que los participantes desde un enfoque sistémico y de co-creación, incorporarán los principios y criterios del ecodiseño en la formulación de proyectos que estuviesen alineados con una estrategia eco-sustentable para una región determinada, cuyos resultados tienen potencial de escalamiento y replicabilidad a otras realidades con estrategias regionales o nacionales de similares características.

Desde una interpretación fuera de la acción, y desde un enfoque de diseño de caso único, los autores concluyen sobre los principios, criterios, componentes y medios necesarios para impulsar procesos de eco-innovación guiados por diseño replicables a otros contextos territoriales. La metodología permite crear una metodología y un toolbox para la formulación de proyectos de innovación impulsados por el diseño adaptados al territorio y alineados con las estrategias de innovación y políticas locales del contexto canario, la que puede ser replicable a otros contextos.

\section{Caso de estudio: el Seminario internacional sobre Políticas Públicas de Diseño (julio 2019)}

El presente artículo expone una experiencia de investigación aplicada y co-desarrollada por los autores en el marco de un proceso de formulación de proyectos para su aplicación al programa de cooperación internacional de investigación "Campus América 2019"3 financiado por instituciones públicas y privadas que tiene lugar en Canarias (España). Este programa de cooperación internacional de la Universidad de La Laguna (ULL) tiene como propósito el incremento de la interacción y las relaciones internacionales entre universidades y organismos de investigación que compartan los mismos intereses y líneas de trabajo. Su objetivo es fomentar la transferencia de conocimiento entre la ULL y universidades latinoamericanas para propiciar el debate y la participación en la resolución de los problemas actuales de la sociedad y generar una apuesta conjunta por la innovación (Universidad de La Laguna, 2019).

En este marco, se celebró el Seminario internacional sobre Políticas Públicas de Diseño donde participaron como países invitados Chile y México. Estas jornadas fueron concebidas como un espacio para compartir, reflexionar y debatir sobre las políticas públicas para el fomento del diseño desde una mirada de América hacia Canarias y viceversa. Un encuentro para impulsar acuerdos de colaboración continuada entre los grupos de investigación participantes, así como foro para la cooperación entre universidades latinoamericanas y donde afianzar las relaciones internacionales.

Este Seminario fue el marco escogido para realizar un encuentro entre representantes de los entes de investigación e innovación de Canarias y los actores del diseño canario cuyo 
propósito era realizar propuestas de proyectos de $\mathrm{I}+\mathrm{D}+\mathrm{i}$ impulsados por el diseño y alineados con las políticas de innovación regionales.

\section{Desarrollo de un taller para la investigación en diseño en Canarias}

En el mes de julio de 2019, un conjunto de actores del Ecosistema del Diseño de Canarias se da cita en el Seminario internacional sobre Políticas Públicas de Diseño, para revisar la evolución del diseño en la comunidad autónoma y debatir sobre los retos que debe abordar el diseño como disciplina y actividad, clave para el impulso de la innovación, el desarrollo y la prosperidad socioeconómica, cultural y medioambiental en el Archipiélago. A partir de la colaboración iniciada con el equipo de investigación de la Escuela de Diseño de la Pontifica Universidad de Chile, tras la invitación del investigador anfitrión, la actividad se fue perfilando hacia una propuesta orientada a la acción a través del alineamiento del ecosistema de diseño con los ejes estratégicos del sistema nacional de innovación local. De este modo, los objetivos que se plantearon para el taller fueron dos:

1. Trabajar colaborativamente sobre la realización de posibles proyectos de investigación e innovación a trasladar a la Agencia Canaria de Investigación, Innovación y Sociedad de la Información (ACIISI) por parte de los organismos de investigación y empresas a través de las diversas subvenciones abiertas en este instante en las áreas prioritarias de la estrategia de Especialización Inteligente de Canarias (RIS3), relativos al Fondo Europeo de Desarrollo Regional (FEDER) 2014-202; y

2. Constituir una red para impulsar la creación de una Estrategia Regional de Diseño.

Con estos objetivos se llevó a cabo un taller el cual fue diseñado a partir de las herramientas utilizadas en la experiencia Sistema Diseño Canarias. El diseño como agente de innovación y desarrollo en Canarias ${ }^{4}$, realizada en mayo de 2017 y se incluyeron los aprendizajes que había dejado el proyecto Puente Diseño Empresa $a^{5}$ en el caso chileno. La finalidad del taller fue la formulación de propuestas para incorporar el diseño en la Estrategia de Especialización Inteligente (RIS3) en el eje prioritario de "Crecimiento verde y sostenibilidad", centrándonos en el objetivo específico vinculado a la eco-innovación. Los participantes de esta actividad fueron organizados en diversos grupos de trabajo entre los que se juntaron representantes de entidades públicas regionales, agrupaciones empresariales, diseñadores, investigadores y docentes de diseño. El taller se desarrolló en diferentes fases y se crearon herramientas ad-hoc para sugerir propuestas en torno a la investigación e incentivo del diseño en la economía sostenible (Ver Tabla 1). 


\begin{tabular}{|c|c|c|c|}
\hline Fase & Objetivos & Instrumento & $\begin{array}{l}\text { Documentos de } \\
\text { consulta }\end{array}$ \\
\hline $\begin{array}{l}\text { Focalización de } \\
\text { objetivo }\end{array}$ & $\begin{array}{l}\text { Identificar propuestas para } \\
\text { proyectos de investigación en } \\
\text { diseño que se ajusten a las } \\
\text { prioridades estratégicas de las } \\
\text { RIS3 y a sus objetivos } \\
\text { específicos. Seleccionar } \\
\text { aquellas de mayor viabilidad y } \\
\text { afinidad }\end{array}$ & $\begin{array}{l}\text { - Matriz de } \\
\text { prioridad }\end{array}$ & $\begin{array}{l}\text { - Objetivos RIS3 } \\
\text { - Objetivos RIS3 } \\
\text { específicos } \\
\text { - Análisis DAFO del } \\
\text { Sistema Diseño } \\
\text { Canarias }\end{array}$ \\
\hline $\begin{array}{l}\text { Formalización } \\
\text { de propuestas }\end{array}$ & $\begin{array}{l}\text { Construcción de la matriz de } \\
\text { marco lógico para proyectos } \\
\text { guiados por diseño Formular } \\
\text { propuestas de proyectos de } \\
\text { investigación guiados por el } \\
\text { diseño a partir de un marco } \\
\text { lógico atendiendo a cuestiones } \\
\text { relativas a desafío a resolver, } \\
\text { objetivos, actividades, } \\
\text { identificación de actores } \\
\text { relevantes para el proyecto y } \\
\text { resultados esperados }\end{array}$ & $\begin{array}{l}\text { - Lienzo de } \\
\text { proyecto }\end{array}$ & $\begin{array}{l}\text { - Resumen de la } \\
\text { línea de } \\
\text { financiación } \\
\text { - Instrucciones para } \\
\text { el lienzo de } \\
\text { proyecto }\end{array}$ \\
\hline Sociabilización & $\begin{array}{l}\text { Socializar las propuestas de } \\
\text { proyectos de investigación }\end{array}$ & & \\
\hline
\end{tabular}

Tabla 1. Fase, Objetivos, Instrumento y Documentos de consulta.

\section{Proyectos de innovación impulsados por el diseño como resultado}

Los proyectos de investigación surgidos son dos y guardan relación con una prioridad temática y un eje específico de la RIS3 para Canarias: I) Crecimiento verde y sostenibilidad (Ver Tabla 2 y 3 ). 


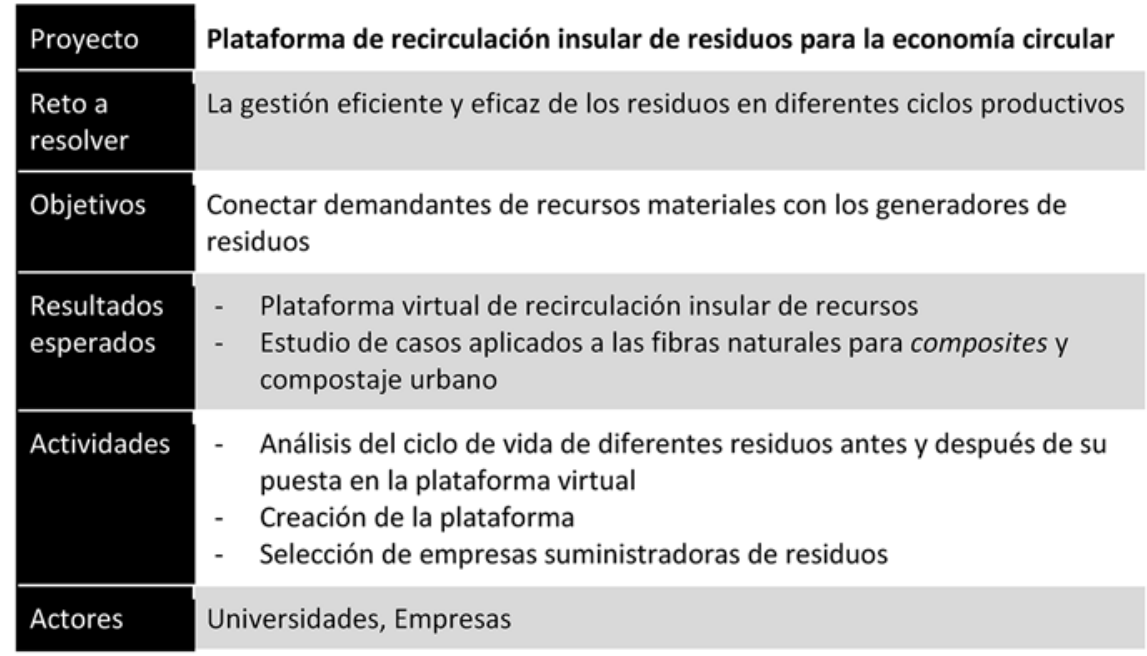

Tabla 2. I.I Eco-innovación, agricultura, pesca y protección del medio ambiente

\begin{tabular}{|c|c|}
\hline Proyecto & Ecodiseño para el sector agroalimentario \\
\hline $\begin{array}{l}\text { Reto a } \\
\text { resolver }\end{array}$ & $\begin{array}{l}\text { Reducir el impacto medioambiental de productos, procesos y servicios del } \\
\text { sector agroalimentario }\end{array}$ \\
\hline Objetivos & $\begin{array}{l}\text { Fomentar la economía circular mediante el análisis y rediseño de productos } \\
\text { y servicios desde un enfoque de Eco-diseño }\end{array}$ \\
\hline $\begin{array}{l}\text { Resultados } \\
\text { esperados }\end{array}$ & $\begin{array}{l}\text { - Plan formativo para diseñadores de productos y servicios, y agentes del } \\
\text { sector agroalimentario en metodologías de Eco-diseño } \\
\text { - Realización de proyectos de investigación y desarrollo públicos- } \\
\text { privados para el desarrollo de productos y servicios eco-innovadores } \\
\text { en el sector agroalimentario }\end{array}$ \\
\hline Actividades & $\begin{array}{l}\text { - Diseño de un plan formativo en Eco-diseño para el sector } \\
\text { agroalimentario } \\
\text { - Trabajos conjuntos de análisis del ciclo de vida de productos y servicios } \\
\text { agroalimentarios para su rediseño mediante metodologías de Eco- } \\
\text { diseño que sirvan de ejemplo de buenas prácticas al sector } \\
\text { - Difusión de casos de buenas prácticas y resultados }\end{array}$ \\
\hline Actores & pos de investigación), pymes y administración \\
\hline
\end{tabular}

Tabla 3. I.II Eco-innovación, agricultura, pesca y protección del medio ambiente. 


\section{Discusión de los resultados}

En el caso canario expuesto, la aplicación de la metodología propuesta ha posibilitado la formulación de dos proyectos de investigación e innovación de diseño asociados a la sostenibilidad y el crecimiento verde como campos del conocimiento prioritario para la región. Dado que este es un caso de investigación aplicada en un contexto particular, para una generalización o consolidación de la propuesta, este debe ser testeado en otras regiones. Por otro lado, el bagaje previo de los autores en contextos diferenciados pero bajo una misma temática de estudio, el propio estudio de casos y su posterior puesta en común de las conclusiones extraídas en cada uno de ellos ha servido de hipótesis para afirmar que los proyectos de impulso del uso del diseño en un territorio deben ser orientados por un modelo instrumental que pueda alinear la acción del diseño con los ejes estratégicos de las políticas públicas locales. Este hecho pone de manifiesto la importancia y necesidad de continuidad que deben tener los programas de cooperación internacional de investigación auspiciados por las instituciones públicas.

Desde una reflexión en la acción, los instrumentos creados ad hoc para el taller, habilitaron a los participantes del ecosistema de diseño canario en la identificación de oportunidades, necesidades y propuestas de solución deseables, factibles y viables para mejores escenarios futuros. Permitieron a los participantes identificar potenciales proyectos impulsados por el diseño y alineados específicamente a las convocatorias públicas competitivas de financiación de la investigación y de la innovación, todo ello, ajustándose a los ejes prioritarios estratégicos de desarrollo y competitividad de un territorio.

Desde una interpretación fuera de la acción, mediante la sistematización de la experiencia, los autores determinan que los principios y criterios que pueden impulsar un proceso de eco-innovación guiado por diseño son:

- El diseño como factor de innovación facilita el cambio hacia soluciones sostenibles teniendo en cuenta los impactos medioambientales a lo largo de todo su ciclo de vida.

- El diseño es un elemento clave en la generación de una economía regenerativa ya que incide en el desarrollo de los diferentes tipos de eco-innovación: de productos, de procesos, de organización, de comercialización, de flujo de materiales y social.

- La utilización del diseño como motor de desarrollo en un territorio determinado mejora la productividad y la competitividad económica, social y medioambiental de manera sostenible.

Del mismo modo, los autores determinan que la metodología o toolkit debe considerar tres fases y los siguientes componentes:

1. Focalización en los propósitos que se presentan en las convocatorias, mediante una lluvia de ideas y posterior selección de aquellas ideas de mayor viabilidad y afinidad.

2. Formalización de la iniciativa en una propuesta que surja como resultado del alineamiento de las expectativas del ecosistema de actores con los propósitos de la convocatoria, mediante la construcción de un marco lógico co-creado.

3. Sociabilización del proyecto con los stakeholders pertinentes para lograr el apoyo político a la iniciativa presentada mediante un breve peach. 
Este marco lógico considera los siguientes componentes:

- el desafío a resolver,

- los objetivos para el desafío

- las actividades necesarias

- la identificación de actores relevantes para el proyecto,

- los resultados esperados

Finalmente, los autores sostienen que para el desarrollo de procesos de eco-innovación guiado por diseño, el conjunto de principios, criterios, fases y componentes requieren de, al menos, dos instrumentos claves:

- Matriz de prioridad: lienzo que categoriza la afinidad de las ideas surgidas como proyectos de investigación con la viabilidad de las líneas estratégicas de la convocatoria competitiva pública de financiación de proyectos. Las propuestas son organizadas de acuerdo a su viabilidad e impacto.

- Lienzo de proyecto: lienzo que define en detalle los elementos necesarios para establecer un plan de investigación. Esta herramienta ayuda a definir con mayor detalle y precisión el proyecto a desarrollar, estableciendo sus objetivos, actividades, resultados esperados, entre otra información (Ver Figuras 1a y b).

1a.

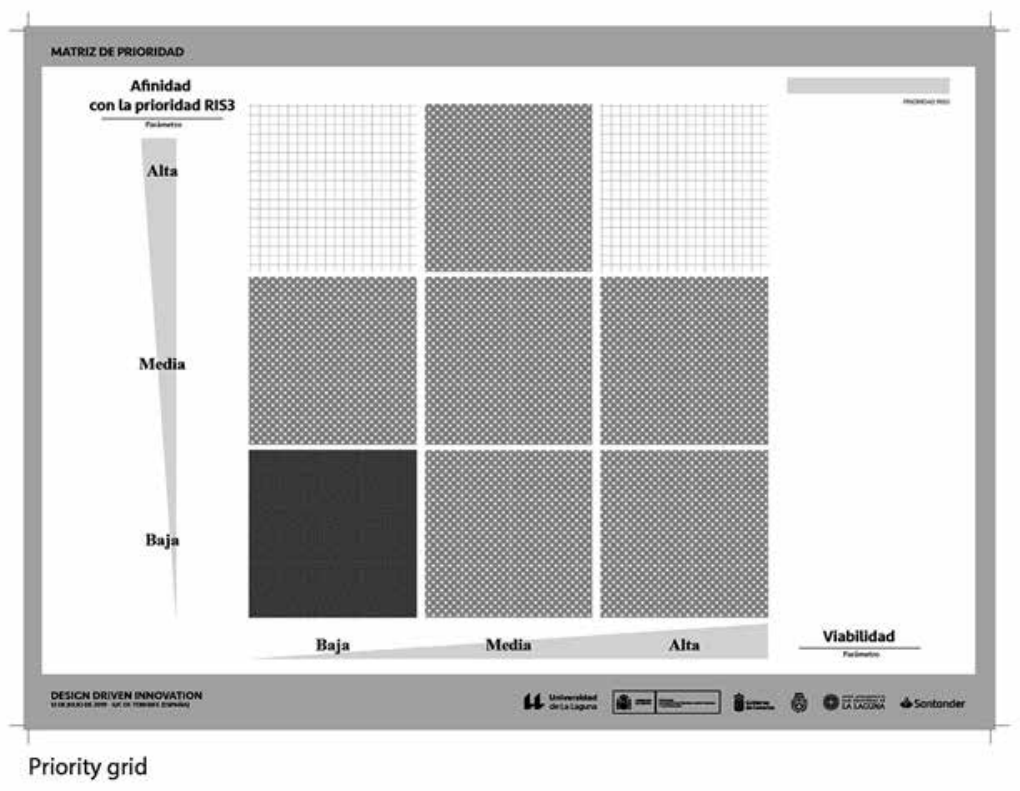




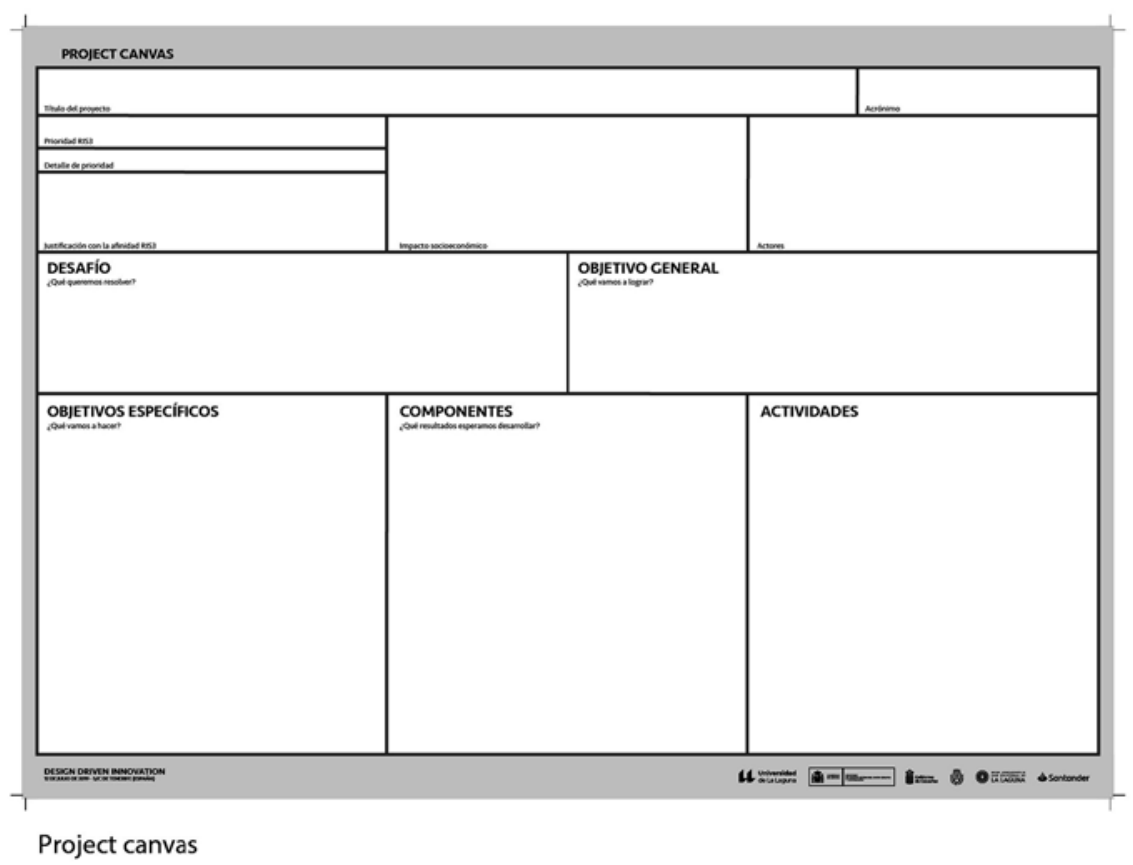

$1 b$.

Figura 1a (p. 274) y b. Herramientas utilizadas en el taller de proyectos de innovación impulsados por el diseño (Desarrollado por Bernardo Candela San Juan y Katherine Mollenhauer).

\section{Conclusiones}

La experiencia presentada, si bien tiene una localización particular, sus conclusiones permiten lograr un conocimiento respecto de aquellos elementos comunes que deben ser considerados como parte de una metodología y un toolkit que facilite el alineamiento de la investigación impulsada por el diseño con las políticas territoriales de I+D+i.

Este hecho permite que, con el apoyo de entidades de transmisión del conocimiento, el sector empresarial -formado principalmente por pymes con escasa capacidad estratégica y de innovación- estas propuestas puedan beneficiarse del sistema regional de investigación e innovación de un territorio.

La realización del taller, permitió que, desde una interpretación fuera de la acción y desde un enfoque de diseño de caso único, los autores crearan una metodología y un toolkit para la formulación de proyectos de innovación impulsados por el diseño adaptados al territo- 
rio y alineados con las estrategias de innovación y políticas locales del contexto canario la cual puede ser replicable a otros contextos.

Con estos resultados los autores esperan contribuir a la generación de conocimiento propio del diseño en el ámbito de las políticas públicas y de las estrategias para el desarrollo de los sistema diseño de diversos países y regiones.

\section{Notas}

1. The Bureau European Designs Associations (BEDA) es una organización sin fines de lucro que cuenta con 47 miembros de unos 25 estados miembros en Europa. Los miembros de BEDA son centros de promoción de diseño y otras organizaciones financiadas con fondos públicos que promueven el diseño a nivel nacional o regional, así como asociaciones profesionales y comerciales para diseñadores de toda Europa. Fundada en 1969, su visión es que el diseño se impulse en Europa como motor del crecimiento sostenible y la prosperidad. Next generation design policy for Europe (2019): https://dl.dropboxuser content.com/s/sw17dwwt55bs1eh/Next\%20generation\%20design $\% 20$ policy\%20for $\% 20$ europe $\% 20$ Nov\%202019.pdf?dl=1

2. Los cuatro grandes ámbitos de acción del diseño alineados con las prioridades de la Comisión Europea son: I) Ecología: Diseño para la economía climática-neutra, II) Economía: Garantizar la prosperidad para todos a través de la colaboración mediante la innovación impulsada por el diseño, III) E-Europa: Diseño digital y datos, y IV) Ethos: Diseño para la democracia, los valores y las relaciones internacionales.

3. Campus América es un proyecto internacional de la Universidad de La Laguna (ULL) que se celebra de forma bianual y que cuenta con el apoyo y la participación del Gobierno de Canarias, Cabildo de Tenerife y Ayuntamiento de San Cristóbal de La Laguna. Campus América se celebra cada dos años y responde al interés de la sociedad canaria en mantener e intensificar las relaciones con América.

4. Sistema Diseño Canarias. El diseño como agente de innovación y desarrollo en Canarias fue un taller realizado en mayo del 2017 en el marco de una investigación doctoral titulada "Sistema Diseño Canarias. Aportaciones para una política de diseño para la comunidad autónoma de Canarias" cuyo objetivo fue testear la formulación del Sistema Diseño Canarias (SDC). En el taller se realizaron tres actividades participativas para proponer políticas de diseño: 1) mapeo de agentes e interacciones, 2) análisis DAFO, 3) codesarrollo de propuestas politicas públicas adaptadas al sistema regional de diseño.

5. Página web del proyecto Puente Diseño-Empresa https://www.puentedisenoempresa.cl/

\section{Referencias Bibliográficas}

Alarcón Castro, J. (2011). Gestión del Diseño. Presentación digital. 
Álvarez, M. J.; Fernández, R. I. y Romera, R. (2014). ¿Es la eco-innovación una estrategia inteligente de especialización para Andalucía? Una aproximación desde el análisis multivariante. Revista de Estudios Regionales, (100),171-195. [fecha de Consulta 9 de Noviembre de 2020]. ISSN: 0213-7585. Disponible en: https://www.redalyc.org/articulo. oa? $\mathrm{id}=755 / 75533716007$

Carrillo-Hermosilla, J.; Del Río P. y Könnlä, T. (2010). Diversity of eco-innovations: Reflections from selected case studies, Journal of Cleaner Production 18, 1073-1083.

Chisolm, J.; Mortati, M. y Villari, B. (2013). Glossary. Describing the system of European design policy.

Comisión Europea (2010). Política regional que contribuya al crecimiento inteligente en Europa 2020. COM (2010) 553 final. Bruselas

Comisión Europea (2011). Innovación para un futuro sostenible. Plan de Acción sobre Ecoinnovación (Eco-AP) COM(2011) 899 final. Bruselas https://eur-lex.europa.eu/legal-content /ES/TXT/PDF/?uri=CELEX:52011DC0899\&from=RO (último acceso 01/11/2020)

Comisión Europea. (2013). Aplicación de un plan de acción para la innovación en materia de diseño Translation. Original document is in English SWD(2013) 380. Bruselas.

Díaz-García, C.; González-Moreno, A. y Sáez-Martínez F. J. (2015). Eco-innovation: insights from a literature review, Innovation: Management, Policy \& Practice, 2015.

El Plan de Acción sobre Ecoinnovación - Plan de Acción sobre Ecoinnovación - Comisión Europea. (2020). https://ec.europa.eu/environment/ecoap/about-action-plan/objectivesmethodology_es (último acceso 09/11/2020).

Finland, M. of E. and the E. (2013). Design Finland Programme, 1-105.

Freeman, C. (1995). The 'National System of Innovation' in historical perspective, Cambridge Journal of Economics 19 (2), pp. 5-24.

Halila, F., y Rundquis, T. J. (2011). The development and market success of environmental innovations: a comparative study of environmental innovations and "other" innovations in Sweden. European Journal of Innovation Management, 14 (3), 278-302.

Hobday, M.; Boddington, A. y Grantham, A. (2012). Policies for design and policies for innovation: Contrasting perspectives and remaining challenges. Technovation, 32(5), 272-281.http://doi.org/10.1016/j.technovation.2011.12.002 (último acceso 01/08/2017)

Irwin, T.; Tonkinwise, C. y Kossoff, G. (2015). Transition design: A proposal for a new area of design practice, study, and research. School of Design, Carnegie Mellon University. Design and Culture.

Johansson, G. y Magnusson, T. (1998). Eco-innovations: a novel phenomenon? Journal of Sustainable Product Design, 7, 7-18.

Kuehne, C. (2007). Regional Instruments, The ECREIN experiences and regional aspects of financing eco-innovation, Ponencia presentada al 9th ETAP Forum on eco-innovation.

Love, T. (2005). Design Infrastructure : Australian Developments. En 2005IDC New Design Paradigms (pp. 1-11). Douliou, Taiwan.: International Association of Societies of Design Research.

Love, T. (2007). System dynamics modelling of national design infrastructure development. In K. Fielden \& J. Sheffield (Eds.), Systemic development: local solutions in a global environment. (pp. 1-22). Auckland. 
Lundvall, B-A. (1985). 'Product Innovation and User-Producer Interaction', Aalborg University Press, Denmark.

Machiba, T. (2010). Eco-innovation for enabling resource efficiency and green growth: development of an analytical framework and preliminary analysis of industry and policy practices. International Economics and Economic Policy, 7 (2), 357-370.

Mollenhauer Gajardo, K. A. (2007). Sistema de Innovación Design-driven. Modelo instrumental para un desarrollo tecnológico culturalmente sostenible. Universidad de Barcelona, Barcelona (España).

Moultrie, J. y Livesey, F. (2009). International Design Scoreboard: Initial indicators of international design capabilities. Design Council, London.

OCDE (2009). Eco-innovation in industry: enabling Green Growth, Paris, Published by OECD.

OECD (2014). Hacia una mejora de políticas para la ecoinnovación, UAM, México, https:// doi.org/10.1787/9789264219267-es (último acceso 05/11/2020).

O’Ryan, R. y Schaper, M. (2017). Marco conceptual. In S. Rovira, J. Patiño \& M. Schaper, Ecoinnovación y producción verde. Una revisión sobre las políticas de América Latina y el Caribe (1st ed., pp. 13-17). Santiago: Naciones Unidas.

Raulik-Murphy, G. y Cawood, G. (2009). "National Design Systems" - a tool for policy-making. En Creative industries and regional policies: making place and giving space (pp. 23-24). Birmingham, United Kingdom.

Rennings, K. (2000). Redefining innovation - eco-innovation research and the contribution from ecological economics. Ecological Economics 32 (2000) 319-332.

Schiederig, T.; Tietzer, F. y Herstatt, C. (2012). Green innovation in technology and innovation management - an exploratory literature review, RङD Management, 42, 180-192.

Spencer, J. (2018). Design for dynamic challenges: key attributes of designers for leading interdisciplinary research and projects. Diseña (13), 84-109. Doi:10.7764/disena.13.84-109

Sun, Q. (2010). Design Industries and Policies in the UK and China: A Comparison. Design Management Review, 21(4), 70-77. http://doi.org/10.1111/j.1948-7169.2010.00097.x (último acceso 06/07/2017)

Swann, G. M. P. (2010). The Economic Rationale for a National Design Policy. Londres.

Universidad de La Laguna. (2019). Campus América 2019. https://www.ull.es/portal/campus america/ (último acceso 26/11/19).

Vicente, M. A. y Tamayo, U; (2014), Estudio Temático de Casos Innobasque “Ecoinnovación”.

Whicher, A. (2014). Mapping Design Innovation Ecosystems Design. See Platform Bulletin, (12), 4-7.

Whicher, A. (2016). Benchmarking Design for Innovation Policy in Europe. Cardiff Metropolitan University.

Whicher, A. y Cawood, G. (2012). // European Design Systems and Innovation Policy. Cardiff, Reino Unido.

Whicher, A.; Harris, C.; Beverley, K. y Swiatek, P. (2017). Design for Circular Economy: Developing an Action Plan For Scotland. PDR at Cardiff Metropolitan University.

World Design Summit Organization Inc. (2017). Montréal Design Declaration (p. 7). Montréal: Design Summit Meeting Steering Committee. https://worlddesignsummit. com/wp-content/uploads/2017/10/20171004_WDSM2017_livret-40pages_declaration _65X9_AN-1.pdf (último acceso 14/06/18). 


\begin{abstract}
Currently, an innovation process is not understood that does not take into account the economic, social and environmental impact that may derive from its implementation. Design as an agent of innovation in sociocultural practices can facilitate the change and transformation necessary for the future to be sustainable, fair and prosperous for the planet and its communities. However, identifying the contribution of the design requires determining the principles, criteria and methodological components that must be activated, and putting them into action through a method that addresses the process co-creatively, from the identification of the problem or opportunity, the ideation of the possible solution answers until the award of competitive funds for the implementation of the project.

The research was carried out in the context of an international research cooperation program, funded by public and private institutions, which generated proposals for design-driven research projects, specifically aligned with the priority axis "Green growth and sustainability" of the Strategy. Smart Specialization of the Canary Islands 2014-2020. The authors systematized the results from an applied research and single-case design perspective and as a result present a methodology and a toolkit for aligning design-driven research with territorial research and innovation policies.

Finally, the authors, based on the results of the application, conclude that the development of a methodology that allows the alignment and activates the potential contributions of the design ecosystem with a specific territorial innovation system, has the benefits of expanding the opportunities for action of discipline, at the same time as contributing to the achievement of the strategic objectives for the sustainable development of the region.
\end{abstract}

Keywords: Design for Eco-Innovation - Design Ecosystem - Design Methodology - Design-guided Innovation Policies - Participatory Methodology - Local Innovation Ecosystem - Systemic Approach.

Resumo: Atualmente não há entendimento de um processo de inovação que não leve em consideração os impactos econômicos, sociais e ambientais que podem decorrer de sua implementação. $\mathrm{O}$ design como agente de inovação nas práticas socioculturais pode facilitar as mudanças e transformações necessárias para que o futuro seja sustentável, justo e próspero para o planeta e suas comunidades. No entanto, identificar a contribuição do design requer determinar os princípios, critérios e componentes metodológicos que devem ser ativados, e colocá-los em ação por meio de um método que trate o processo de forma cocriativa, a partir da identificação do problema ou oportunidade, da ideação de a solução possível responde até a atribuição de verbas competitivas para a implementação do projeto.

A investigação foi realizada no âmbito de um programa de cooperação internacional em investigação, financiado por instituições públicas e privadas, que gerou propostas de projectos de investigação orientados para o design, especificamente alinhados com o eixo prioritário "Crescimento verde e sustentabilidade" da Estratégia Especialização inteligente das Ilhas Canárias 2014-2020. Os autores sistematizaram os resultados de uma perspectiva de pesquisa aplicada e design de caso único e, como resultado, apresentam uma metodolo- 
gia e um kit de ferramentas para alinhar a pesquisa orientada para o design com políticas territoriais de pesquisa e inovação.

Por fim, os autores, com base nos resultados da candidatura, concluem que o desenvolvimento de uma metodologia que permita alinhar e ativar os potenciais contributos do ecossistema do design com um sistema de inovação territorial específico, tem os benefícios de ampliar as oportunidades de ação de disciplina, ao mesmo tempo que contribui para a concretização dos objetivos estratégicos para o desenvolvimento sustentável da região.

Palavras chave: Design para Ecoinovação - Ecossistema de Design - Metodologia de Design - Políticas de Inovação Orientadas por Design - Metodologia Participativa - Ecossistema de Inovação Local - Abordagem Sistêmica. 